\title{
Nanoparticles to Nanoholes: Fabrication of Porous GaN with Precisely Controlled Dimension via the Enhanced GaN Decomposition by Au nanoparticles
}

\author{
Puran Pandey ${ }^{l}$, Mao Sui ${ }^{l}$, Ming-Yu Li ${ }^{l}$, Quanzhen Zhang ${ }^{1}$, Sundar Kunwar ${ }^{l}$, Jiang $W u^{2,3}$, \\ Zhiming M. Wang ${ }^{3}$, Gregory. J. Salamo ${ }^{4}$, and Jihoon Lee $e^{1,4^{*}}$ \\ ${ }^{1}$ College of Electronics and Information, Kwangwoon University, Nowon-gu Seoul 01897, \\ South Korea \\ ${ }^{2}$ Department of Electronic and Electrical Engineering, University College London, \\ Torrington Place London, WC1E 7JE, UK \\ ${ }^{3}$ Institute of Fundamental and Frontier Sciences, University of Electronic Science and \\ Technology of China, Chengdu 610054, P.R. China \\ ${ }^{4}$ Institute of Nanoscale Science and Engineering, University of Arkansas, Fayetteville AR \\ 72701, USA
}

\footnotetext{
ABSTRACT: Porous GaN exhibits unique optoelectronic, chemical and physical properties such as shift of band gap, increased surface area ratio, excellent chemical, mechanical and thermal stability as well as efficient luminescence as compared to its bulk counterpart. Herein, we demonstrate a precise, efficient and still cost effective method of the fabrication of porous $\mathrm{GaN}$ through the enhanced $\mathrm{GaN}$ decomposition by using $\mathrm{Au}$ nanoparticles (NPs) as a catalyst, in which the size, density and shape of the pores (nanoholes, NHs) can be precisely controlled. By the thermal annealing assisted with the Au NPs, the NHs are successfully fabricated and the existence of Au NPs significantly accelerate the GaN decomposition at the interface between the NPs and GaN due to the $\mathrm{Ga}$ absorption by the Au NPs. We systematically study the formation mechanism of NHs assisted by the Au NPs by means of annealing temperature, duration and Au deposition amount and the results are systematically analyzed and discussed.
} 


\section{口 INTRODUCTION}

Due to its unique physical properties such as enlarged surface area ratio, efficient luminescence, and shift of band gap as well as the excellent chemical, mechanical and thermal stability, porous $\mathrm{GaN}$ has been successfully adapted in various applications. ${ }^{1-5}$ The properties of porous $\mathrm{GaN}$ are significantly affected by the variation of the morphology of pores, such as size, density and shapes. ${ }^{6-8}$ For example, the control of the refractive index of $\mathrm{GaN}$ can be key element for designing the optical devices in which the refractive index decrease with the increased pores density, investigated by the prism coupling technique, ${ }^{6}$ and the properties of $\mathrm{GaN}$ overgrown on porous $\mathrm{GaN}$ was improved with the smaller pore size. $^{7}$ Porous GaN has been utilized in several applications such as sensors, ${ }^{9-10}$ LEDs, ${ }^{11-13}$ as well as templates for the lattice-mismatched heteroepitaxy. ${ }^{14-16}$ For instance, in the case of hydrogen gas sensor, significantly improved sensitivity with the porous GaN was reported due to much increased surface area ratio of the Pd/porous GaN interface. ${ }^{9}$ The porous $\mathrm{GaN}$ with various size, shape and high density of pores enhance the blue shift luminescence as well as exhibits the highest level of sensitivity in hydrogen sensor. ${ }^{10}$ Also, utilizing the porous GaN can significantly increase the light extraction and internal quantum efficiency of LEDs due to the enhanced light scattering by the pores. ${ }^{11-13}$ While the high defect density of GaN grown on sapphire can cause the degradation in the efficiency of the GaN based LEDs,${ }^{17}$ porous GaN can provide an intermediate layer for high quality epitaxial GaN growth by releasing the strain and reducing the dislocation density of the overgrown GaN. ${ }^{14-16}$ The fabrication of the porous $\mathrm{GaN}$ has been usually done by the electrochemical or chemical etching ${ }^{18-20}$ and they have found their own successes. However, the precise control of the size, shape and density of the pores has been 
ever challenging and the various chemicals and complex procedures utilized have been potential threats to the performance of the devices based on it. In this work, we report a unique method for the fabrication of porous $\mathrm{GaN}$ of a precise and rather simple approach through the enhanced decomposition of GaN by utilizing Au NPs as a catalyst. The pores (nanoholes, NHs) are successfully fabricated on GaN owing to the concurrent occurrence of several phenomena such as natural GaN decomposition, release of nitrogen as $\mathrm{N}_{2}$ gas, formation of Au-Ga alloy NPs, enhanced penetration of NPs, and the evaporation of AuGa NPs. The resulting morphology of the NHs is strictly inherited from the Au NPs. Depending up on the systematical control of annealing temperature, duration and the size and density of Au NPs, the shape, size and density of NHs can be correspondingly controlled.

\section{— EXPERIMENTAL SECTION}

Materials. The fabrication of porous $\mathrm{GaN}$ was performed on the $c$-plane $\mathrm{GaN}$ (0001) template grown on sapphire. (Technologies and Devices International (TDI), Inc., USA) The epitaxial-ready GaN were semi-insulating with a thickness of $\sim 10 \mu \mathrm{m}$. Figure S1 shows the reflectance of bare GaN (0001) that demonstrate the cut-off wavelength of $\mathrm{GaN}$ was observed at $364.5 \mathrm{~nm}(\sim 3.4 \mathrm{eV}$ bandgap of $\mathrm{GaN})$ with the characteristic oscillation pattern caused by the interference of light reflected at both $\mathrm{GaN}$ and sapphire surfaces. After the preparation of samples, they were soldered on a sample holder using indium and degassed at $700{ }^{\circ} \mathrm{C}$ for 30 min with the ramping rate of $2{ }^{\circ} \mathrm{Cs}^{-1}$ in a pulsed laser deposition $(\mathrm{PLD})$ chamber under $1.0 \times 10^{-4}$ Torr. The clean surface morphology of GaN (0001) was observed after degassing. 
Fabrication of Au NPs and NHs. After the degassing, the samples were ready for the $\mathrm{Au}$ deposition. Initially, as a catalyst, various amount of $\mathrm{Au}$ was deposited on $\mathrm{GaN}$ (0001) with a deposition rate of $0.05 \mathrm{~nm} / \mathrm{s}$ at the ionization current of $3 \mathrm{~mA}$ under $1.0 \times 10^{-}$

${ }^{1}$ Torr in a plasma ion-coater chamber. Figure 1a shows the $3 \mathrm{~nm}$ Au deposited GaN sample that demonstrate the uniform distribution of Au with a height distribution of $\pm 2 \mathrm{~nm}$ along with the surface steps. After that, the annealing of sample was carried out with the uniform ramping rate of $2{ }^{\circ} \mathrm{Cs}^{-1}$ under $1.0 \times 10^{-4}$ Torr in the PLD chamber. After performing the annealing for a specified duration, the temperature was quenched down immediately to the ambient temperature in order to prevent the undesired Ostwald ripening. To investigate the temperature effect on the formation of $\mathrm{NHs}, 4 \mathrm{~nm}$ of $\mathrm{Au}$ was deposited and the annealing duration was fixed for $450 \mathrm{~s}$. In order to observe the effect of annealing duration for the evolution of NHs from NPs, the duration was systematically varied between 900 and 3600 s and annealed at $800{ }^{\circ} \mathrm{C}$ while the Au deposition amount (DA) was fixed at $3 \mathrm{~nm}$. For the precise control of shape, size and density of NHs, the temperature was fixed at $900{ }^{\circ} \mathrm{C}$ for the Au DA of 2, 3, 4, 7, 10 and $25 \mathrm{~nm} .950^{\circ} \mathrm{C}$ was selected for the larger Au DA including $5 \mathrm{~nm}$ and the annealing duration was set as $450 \mathrm{~s}$ for all the fabrications.

Characterization. In this research, an atomic force microscope (AFM) with a noncontact mode was employed to characterize the surface morphology of samples (XE70, Park Systems Corp., South Korea). The probes utilized were NSC16/AIBS with a length of $125 \mu \mathrm{m}$, and the tip of the probe has a curvature radius $\left(\mathrm{R}_{\mathrm{c}}\right)$ less than $10 \mathrm{~nm}$ and tip height of $17 \mu \mathrm{m}$. The resonance frequency of the probes are $\sim 270 \mathrm{kHz}$ and the force constant is $40 \mathrm{~N} / \mathrm{m}$. By using XEI software (Park Systems), the obtained data were prepared and analyzed in terms of the AFM top-views, side-views, 2-D Fourier filter transform (FFT) 
power spectra, and cross sectional line-profiles. A scanning electron microscope (SEM) was employed for observing surface morphology in larger scale (CX-200, COXEM, South Korea) and an energy-dispersive X-ray spectroscope (EDS) was utilized for elemental characterization and spectral mapping (Noran System 7, Thermo Fisher, USA). To observe the morphology variation for the fabricated porous GaN, Raman spectra were obtained with a charge coupled device (CCD) detector and the excitation laser wavelength was 532 nm (UNIRAM II, UniNanoTech, South Korea).

\section{— RESULTS AND DISCUSSION}

Behaviors of Au with temperature. Figure 1 illustrates the evolution of Au nanoparticles (NPs) to nanoholes (NHs) on $\mathrm{GaN}(0001)$ at various annealing temperature from 450 to $900{ }^{\circ} \mathrm{C}$ with $3 \mathrm{~nm}$ of Au deposition. Initially, the as-deposited surface showed a flat morphology with a height distribution of $\pm 2 \mathrm{~nm}$ along with the atomic steps as presented in Figure 1a. During the annealing of as-deposited sample, Au NPs were formed based on the Volmer-Weber growth model, ${ }^{21}$ and evolved into various size and shape at the temperature range between 450 and $750{ }^{\circ} \mathrm{C}$. At a relatively lower annealing temperature of $450{ }^{\circ} \mathrm{C}$, the nucleation of caterpillar-shaped NPs was observed owing to the surface diffusion and collision between Au adatoms driven by the thermal energy as shown in Figure $1 \mathrm{~b}$. The surface diffusion coefficient $D$ of the adatoms obeys a scaling relation with the annealing temperature as below: ${ }^{22}$

$$
D \propto \exp \left(-E_{A} / K_{B} T\right),
$$


where $E_{A}$ is the surface diffusion barrier, $K_{B}$ is the Boltzman constant and $T$ is the annealing temperature. Based on the diffusion coefficient $D$, the diffusion length $L_{d}$ can be expressed as:

$$
L_{d}=\sqrt{D \tau},
$$

where $\tau$ is the residual time of adatoms. Inferred from the two equations above, the diffusion length $L_{d}$, proportional to the annealing temperature i.e. the distance an adatom can travel on the surface, can be lengthened by the increased temperature. Thus, when the annealing temperature was increased to $750{ }^{\circ} \mathrm{C}$, the nanostructures can grow larger in size and more regular in shape by absorbing nearby Au adatoms and tiny particles until reaching an equilibrium. As a result, the Au caterpillars are evolved into more compact and regular Au hexagons as shown in Figure 1c. Besides, in terms of the NP shape, Au hexagons with flat-toped facet of (111) were formed rather than semi-spheres in order to minimize the surface energy. ${ }^{23-24}$ The formation of the crystalline Au NPs can be a way to lower the surface energy processed by the NPs. Thus, the Au hexagons possess lower miller index facets such as $\{111\}$ and $\{100\}$. And when the temperature was increased to $900{ }^{\circ} \mathrm{C}$, hexagonal nanoholes (NHs) were formed on the surface as clearly evidenced by AFM topand side-views in Figure 1d,d-1. The formation of the NHs on GaN can be attributed to the enhanced decomposition of $\mathrm{GaN}$ followed by the concurrent occurrence of several phenomena: the details will be discussed in the following sections.

Natural GaN decomposition. Figure 2 shows the surface decomposition of GaN (0001) at high annealing temperatures. In general, with the increased annealing temperature between 800 and $900{ }^{\circ} \mathrm{C}$, tiny pits and Ga droplets were observed. The GaN (0001) surface is dominated by the terraces and steps which corresponds to the layers consisting $\mathrm{Ga}$ and 
$\mathrm{N}$ atoms,${ }^{25}$ as clearly shown in Figure $2 \mathrm{a}$. As seen in the height distribution histogram in Figure 2a-2, the height of the surface steps is mainly distributed within the range of $\pm 1 \mathrm{~nm}$. In general, wurtzite substrate has a high thermal stability owing to the polarity of crystalline structure. Nonetheless, at relatively higher temperatures, GaN undergoes thermal decomposition. ${ }^{26-27}$ Based on the GaN crystal lattice structure, the distance between two neighboring nitrogen atoms in lattice is $3.2 \AA$ and is $1.07 \AA$ for $\mathrm{N}_{2}$ molecule in gas form. Due to the strong holding force of the structural unit of the atoms in $\mathrm{GaN}$, it is hard to predict the release of nitrogen atoms from the solid $\mathrm{GaN}$ as an $\mathrm{N}_{2}$ molecule at low temperature. ${ }^{28}$ However, at high temperature, GaN will be decomposed following the mechanistic desorption as:

$$
\mathrm{GaN}_{(\mathrm{s})}=\mathrm{Ga}_{(\mathrm{l})}+\frac{1}{2} \mathrm{~N}_{2(\mathrm{~g})}
$$

It is obvious from above equilibrium relation that, liquid phase Ga rich nuclei are formed and the nitrogen gas is released. In our case, at $800{ }^{\circ} \mathrm{C}$ annealing for an hour, numerous tiny $\mathrm{Ga}$ droplets appeared on the surface in Figure $2 \mathrm{~b}$, and accordingly the height distribution became slightly wider, i.e. $\pm 2 \mathrm{~nm}$. Due to the Ga-rich condition, the surface diffusion barrier of nitrogen atoms can be significantly reduced. ${ }^{29}$ As demonstrated by Fernandez-Garrido et al., the diffusion barrier calculated for the migration of $\mathrm{N}$ adatoms is $1.4 \mathrm{eV}$ and decreased to $0.2 \mathrm{eV}$ under the Ga-rich condition. Then after annealing at $900{ }^{\circ} \mathrm{C}$ for an hour, as can be expected, the droplets and pits became larger and accordingly the height distribution was increased to $\pm 4 \mathrm{~nm}$ in Figure 2c-2. It implies, the higher annealing temperature in conjunction with the lower diffusion barrier under the Ga-rich condition intensifies the nitrogen atom migration and thus increases the rate of nitrogen desorption, accordingly. In other words, the generated liquid Ga droplets can act as catalysts and 
accelerate the GaN decomposition. In the report of D. D. Koleske et al., the morphology of GaN surface showed Ga residuals surrounded by ravines after a high temperature annealing, which is consistent with above theoretical inference. ${ }^{30}$

From Au nanoparticles to nanoholes. Figure 3 shows the temperature-induced evolution of the NHs from the Au hexagons on the GaN (0001) with the identical $4 \mathrm{~nm} \mathrm{Au}$ deposition and $450 \mathrm{~s}$ annealing. In general, Au NPs act as a catalyst for the formation of $\mathrm{NHs}$ and deeper NHs were fabricated at higher temperature. Initially, at $700{ }^{\circ} \mathrm{C}$, hexagonal Au NPs were fabricated as shown in Figure 3a. When the annealing temperature was increased to 900 and $950^{\circ} \mathrm{C}$ as clearly shown in Figure 3b,c, the NHs were formed on GaN utilizing Au hexagon as a catalyst such that the shape of the NHs were also hexagonal. Meanwhile, at $1000{ }^{\circ} \mathrm{C}$, the $\mathrm{NHs}$ were inter-connected and the shape was distorted as shown in Figure 3d. The formation of NHs can be attributed to the significantly enhanced GaN decomposition assisted by the Au NPs, namely the NP penetration into the GaN. The NP penetration can be the combinational consequence of the following processes concurrently occurring: (a) formation of self-assembled Au NPs, (b) natural decomposition of GaN resulting in the Ga rich condition with tiny Ga droplets and $\mathrm{N}_{2}$, (c) Ga vaporization and diffusion, (d) formation of alloy NPs (Au-Ga), (e) enhanced penetration due to the significant decomposition of GaN at the interface of NPs and GaN, and (f) evaporation of alloy NPs. As mentioned in the previous section, at high temperature above $800{ }^{\circ} \mathrm{C}, \mathrm{GaN}$ can decompose and this leads to the Ga-rich condition, which in turn intensifies the nitrogen decomposition. At the same time, Ga adatoms can vaporize as well as migrate on the surface. With the existence of the Au NPs, the diffused Ga adatoms can now incorporate into the Au NPs, resulting in the formation of the Au-Ga alloy NPs. The Au NPs can absorb 
the elements with an enhanced thermal energy, forming alloy particles on various semiconductors. ${ }^{31-32}$ As a result, significant GaN decomposition can be expected at the interface of GaN and NPs, Ga being absorbed into the Au NPs making Au-Ga alloy NPs. Consequently, the $\mathrm{N}_{2}$ gas release from the $\mathrm{GaN}$ and the $\mathrm{Au}-\mathrm{Ga}$ alloy drill down inside the holes. The ability of NPs to penetrate inside the GaN is directly proportional to the amount of Au until the NP saturates. Eventually, the Au-Ga alloy also can evaporate in the temperature range $800-1000{ }^{\circ} \mathrm{C}$ depending upon its composition, ${ }^{33}$ and as a result the NHs can be formed. Initially, Au deposited $\mathrm{GaN}$ was annealed at $700{ }^{\circ} \mathrm{C}$, and hexagonal Au NPs were fabricated based on the Volmer-Weber growth model as shown in Figure 3a. The height and lateral diameter of typical NPs were $\sim 45$ and $\sim 125 \mathrm{~nm}$ as clearly shown by the line-profile in Figure 3e. As the annealing temperature was increased to $900{ }^{\circ} \mathrm{C}$, the hexagonal NPs were penetrated to GaN surface and as a result the hexagonal NHs were fabricated as shown by the AFM images in the Figure $3 \mathrm{~b}$. The lateral diameter was almost identical to that of the NPs and the depth of NHs was $\sim 37 \mathrm{~nm}$ as clearly shown by the lineprofile. It is clearly demonstrated that the shape, size and density of the NHs were well controlled by that of the NPs. The penetration depth of the NHs can be significantly affected by the environment during the NP penetration. For example, under the same duration, the rate of GaN decomposition can be drastically increased by the increased annealing temperature. Thus, the further rapid decomposition of $\mathrm{GaN}$ at $950{ }^{\circ} \mathrm{C}$ resulted in the increased penetration depth of NHs as shown in Figure 3c. Specifically, the depth of typical NHs formed at $950{ }^{\circ} \mathrm{C}$ was approximately twice of the $\mathrm{NHs}$ formed at $900{ }^{\circ} \mathrm{C}$; i.e. from $\sim 37$ to $\sim 72 \mathrm{~nm}$. Then, at $1000{ }^{\circ} \mathrm{C}$, the penetration depth was significantly enhanced by the high thermal energy. As shown in Figure 3d, the depth of typical NH was increased 
to $\sim 90 \mathrm{~nm}$ but the shape was distorted due to the enhanced $\mathrm{GaN}$ decomposition. Overall, the line-profiles in Figure 3e confirms the lateral diameter of the NP was almost equal to the $\mathrm{NH}$ at different annealing temperatures. Meanwhile, the depth of the $\mathrm{NH}$ was gradually enhanced accordingly with the increased annealing temperature. The FFT power spectra also reflect the surface morphology evolution of the corresponding samples as shown in Figure 3a-3 - d-3. With the formation of hexagonal NPs, the FFT power spectrum also showed the hexagonal shape. Similarly, with the formation of hexagonal NHs and wider range of height distribution, the FFT power spectrum became slightly large hexagonal. Furthermore, with the distorted NHs, the FFT also showed an irregular shape.

Figure 4 shows the energy-dispersive X-ray spectroscopy (EDS) elemental characterization of the NHs. As displayed in Figure 4a, the NHs were formed with $25 \mathrm{~nm}$ Au deposition and annealed at $900{ }^{\circ} \mathrm{C}$ for 3600 s. Figure 4b,c show $\mathrm{Ga}$ and $\mathrm{Au}$ elemental line-profiles of two typical NHs indicated with the green and violet rectangles. Figure 4d,e show the small region elemental spectra of the areas marked by red and blue rectangles. The red rectangle Figure 4d displays a NH with residual inside, while the blue Figure 4e shows a region of GaN surface. The Au M $\alpha 1$ peak at $2.12 \mathrm{keV}$ in EDS spectra is clearly observed as shown with the inset in Figure $4 \mathrm{~d}$ while the $\mathrm{Au} M \alpha 1$ peak was not observed in Figure 4e. The result shows no Au remains on the surface and Au exists inside the NHs after one hour annealing. As shown in Figure $4 \mathrm{~b}$, nearly half of the area of $\mathrm{NH}$ was occupied by the NP residual while no residual was observed inside the NH with the violet rectangle in Figure 4c. This shows that the amount of the remaining Au in the NH is not equal and there is an evaporation of $\mathrm{Au}$ at high annealing temperature. ${ }^{34}$ The evaporation rate $\left(R_{e}\right)$ can be expressed as: ${ }^{35}$ 


$$
R_{e}=\left(3.513 \times 10^{22}\right)\left(T M_{A u}\right)^{-1 / 2} P_{T}
$$

where $T$ is the annealing temperature, $M_{A u}$ is the atomic weight of $\mathrm{Au}$, and $P_{T}$ is the equilibrium pressure of Au vapor at a certain T. According to the equation above, the value of $R_{e}$ can be determined by both $P_{T}$ and $T$. The value of $P_{T}$ is higher at an increased $\mathrm{T}$ and thus the Au evaporation rate can be significantly increased at the elevated temperature. As evidenced by the EDS line-profile in Figure 4b, the Au peak appears at the position where the residual is located. And the rest area shows a high Ga concentration and even higher at the edge of the NHs. This evidences the drastically enhanced decomposition of GaN at the interface between $\mathrm{GaN}$ and $\mathrm{Au}$ NPs as mentioned in the above sections. Figure 4c shows the elemental characterization of a $\mathrm{NH}$ without residual. The inside Ga peak proves the Garich condition and small amount of $\mathrm{Au}$ is still detected after the Au evaporation.

Figure 5 shows the evolution of NHs along with the systematically controlled annealing duration. To investigate the relationship between the $\mathrm{NH}$ formation and annealing duration, the deposition amount and annealing temperature were fixed at $3 \mathrm{~nm}$ and $800{ }^{\circ} \mathrm{C}$ while the duration was varied from 900 to $3600 \mathrm{~s}$. As discussed in the previous section, the natural decomposition of GaN was only slightly occurred at $800{ }^{\circ} \mathrm{C}$ even after one hour without Au NPs. However, with the presence of Au NPs the decomposition was much accelerated at the interface, namely the penetration of NPs into GaN. The penetration depth was gradually increased with the increased annealing duration. Initially, at $800{ }^{\circ} \mathrm{C}$ for $900 \mathrm{~s}$ of annealing, the NPs slightly drilled down the GaN, still showing the NP geometry of the surface due to the insufficient annealing duration and annealing temperature as shown in Figure 5a. The height of typical Au NPs is $\sim 10 \mathrm{~nm}$ as shown by the line profile in Figure $5 \mathrm{~d}$ and is much shorter as compared to the $\sim 45 \mathrm{~nm}$ of NPs in 
Figure 3a. Then, along with the longer annealing duration of $1800 \mathrm{~s}$, the co-existence of small NHs and shorter NPs were observed, as shown in Figure 5b. While the small NPs can completely drill into the GaN, the large ones are still left on the surface, which can be due to the non-uniform size distribution of Au NPs in the preceding sample. The depth of the typical $\mathrm{NH}$ was $\sim 2 \mathrm{~nm}$. At the same time, the remaining comparatively large NPs grew in lateral size accompanying with the increased annealing duration based on the Ostwald's ripening. ${ }^{36}$ Eventually, with the increased annealing duration to $3600 \mathrm{~s}$, the surface was now fully covered by NHs as all Au NPs drilled down into the NHs as shown in Figure 5c. The depth of NHs was $\sim 6 \mathrm{~nm}$ as evidenced by the line-profiles in Figure 5d. (The largescale AFM side-views in the supporting information Figure S2) The results clearly shows that the existence of the Au NPs enhances the decomposition of GaN at the interface between NPs and GaN as the naturally decomposed GaN showed tiny pits and Ga droplets along with the atomic steps under the identical condition, $3600 \mathrm{~s}$ at $800{ }^{\circ} \mathrm{C}$ in Figure $2 \mathrm{~b}$.

Figure 6 shows the map of $\mathrm{NH}$ fabrication as function of annealing temperature and duration. As seen, depending on the fabrication condition, the overall evolution is divided into three regimes: NPs, transition and NHs. In the NP regime, the Au NPs are fabricated with the various shape and size. These NPs can be systematically utilized as catalyst for the fabrication of NHs by the appropriate control of annealing temperature and duration. The co-existence of NHs and drilled Au NPs are clearly shown in the transition regime. Moreover, the increased annealing temperature and duration results in significantly developed $\mathrm{NHs}$ as shown in $\mathrm{NH}$ regime. The surface area ratio (SAR) of related samples are shown in Figure 6a,b. The SAR is expressed as SAR $=$ (Geometric AreaSurface Area $) /($ Geometric Area $) \times 100[\%]$ to describe the variation of the surface 
area. As seen in Figure 6a, the SAR is gradually increased at increased temperature along with the development of $\mathrm{NH}$ depth. However, accompanying with the longer annealing duration, the SAR shows a discontinuous variation. As seen in Figure 6b, the co-existence of NPs and NHs resulted in the lowest value in comparison with the NPs and NHs, which can be due to the compensation between height and depth. And, as the average depth of NHs was developed with longer annealing duration, the SAR was increased.

\section{Controllable fabrication of NPs and NHs with variation of Au deposition}

amount. In this section, the control of size, density and shape of Au NPs and NHs was systematically investigated with the variation of Au deposition amount as shown in Figures 7 and 8. In general, the morphology of NHs can be systematically controlled by the systematic variation of deposition amount. Initially, the Au NPs can be synthesized with precisely controlled shape, size and density with the variation of deposition amount. And, these Au NPs can act as a catalyst for the precise control of the shape, size and density of the NHs. As represented in Figure 7, the size of NPs including the diameter and height sensitively responds to the variation of $\mathrm{Au}$ deposition amount. With the increased $\mathrm{Au}$ deposition amount, i.e. above $10 \mathrm{~nm}$, due to the hugely increased NP size, the resulted crystalline structure is strongly dependent on the growth kinetics. ${ }^{37}$ As a consequence, additional facets $\{110\},\{210\}$, and other higher Miller index facets were observed with the Au hexagons originally enclosed with $\{111\}$ and $\{100\} .{ }^{38-39}$ With the incremental variation of deposition amount from 4 to $25 \mathrm{~nm}$, the diameter was increased from $\sim 100$ to $1000 \mathrm{~nm}$ by over 10 times and the height from 20 to $300 \mathrm{~nm}$ by over 15 times. Meanwhile the density was decreased from $\sim 4 \times 10^{9}$ to $1 \times 10^{7} \mathrm{~cm}^{-2}$ as a compensation. As a result, the corresponding changes in the size and density of NHs were observed with the increased 
deposition amount, as shown in the AFM top-views in Figure 8. Firstly, with $2 \mathrm{~nm}$ deposition, tiny and merged NHs were formed as shown in Figure 8a,a-1. Due to the dense distribution of NPs, the edge of the NHs appeared blur and irregular. With the addition of $1 \mathrm{~nm} \mathrm{Au}$ deposition i.e. at $3 \mathrm{~nm}$, the NHs began to separate with each other and the individual NH was observed as clearly shown in Figure 8b. And during the gradually increased deposition amount up to $25 \mathrm{~nm}$, the dimension including depth and diameter were gradually increased as evidenced by the AFM images. Specifically, with the deposition amount varied from 2 to $25 \mathrm{~nm}$, the dimension of NHs was expanded by $\sim 23$ times from $58 \mathrm{~nm}$ to $1336 \mathrm{~nm}$ in terms of depth, by 23 times from 35 to $835 \mathrm{~nm}$ in terms of diameter. (The specific values are provided in supporting information Table S1) Meanwhile, the density of NHs was dropped by $\sim 525$ times from $8.4 \times 10^{11}$ to $1.6 \times 10^{9} \mathrm{~cm}^{-2}$. As compared with the fabricated Au NPs shown in Figure 7, it can be concluded that the shape, size and density evolution of NHs was well determined by that of Au NPs. The NHs were regular hexagonal with the deposition amount variation from 3 to $7 \mathrm{~nm}$. However, over $10 \mathrm{~nm} \mathrm{Au}$ deposition, following the formation of multifaceted NPs, the NHs with round shape were fabricated instead of the hexagons. (The Raman spectra of the relevant samples are shown in supporting information Figure S3) In the meantime, the fabrication of the small to large sized, high density to low density, round to hexagonal shaped $\mathrm{NHs}$ on $\mathrm{GaN}$ i.e. porous $\mathrm{GaN}$ can be a good alternative material for the significantly efficient optical devices as the morphology of the NHs can effectively tuned the optical properties. ${ }^{6-8}$

\section{Investigation on the formation process of NHs with large Au deposition}

amount. Figure 9 summarizes the annealing temperature and duration effect on the fabricated NHs. The effect of annealing temperature at fixed Au deposition amount $10 \mathrm{~nm}$ 
and annealing duration $3600 \mathrm{~s}$ are shown in Figure 9a,b. (Corresponding AFM images and EDS spectra are shown in supporting information Figures S4 and S5 respectively) Similarly, the effect of annealing duration with higher deposition $(25 \mathrm{~nm})$ at annealed 900 ${ }^{\circ} \mathrm{C}$ are shown in Figure $9 \mathrm{c}-\mathrm{e}$. (Corresponding AFM images spectra are shown supporting information in Figure S6) The depth and diameter obtained from the AFM data is plotted in Figure 9g,h. In general, the residual Au-Ga alloys can be distinguished as the bright spots in the NHs as shown in the SEM images as the electron beam is more sensitive to the metal. However, at the increased annealing temperature, the bright spots are disappeared in Figure 9a. This can be due to the thermal evaporation of Au-Ga alloy NPs at hightemperature during the annealing as mentioned in the above section. The rate of evaporation can be expected higher at an elevated temperature, i.e. $950{ }^{\circ} \mathrm{C}$ compared to $900{ }^{\circ} \mathrm{C}$. As characterized by the EDS spectra shown in Figure 9a-1,b-1 (Full-range EDS spectra seen in supporting information Figure S7), the Au M $\alpha 1$ peak $(2.12 \mathrm{keV})$ of $900{ }^{\circ} \mathrm{C}$ annealing appeared 3700 counts while the count of $950{ }^{\circ} \mathrm{C}$ was reduced to 2700 by $27 \%$. Meanwhile, the NHs depth and diameter were both increased from 361 to $487 \mathrm{~nm}$ and 660 to $727 \mathrm{~nm}$ respectively. Furthermore, in terms of shape, the NHs are approximately round with clear boundary at $900{ }^{\circ} \mathrm{C}$ while at $950{ }^{\circ} \mathrm{C}$ the irregular shaped $\mathrm{NHs}$ with blur boundary were observed. With $25 \mathrm{~nm}$ of Au deposition, the annealing temperature was set at $900{ }^{\circ} \mathrm{C}$ to protect the shape from the extensive natural decomposition of GaN. After the annealing for $1800 \mathrm{~s}$, the residual of $\mathrm{Au}$ in NHs were witnessed and the NHs appeared in round shape while the residuals appear irregular shape connected with random edges of particular NHs. When the duration was increased to $3600 \mathrm{~s}$, the amount of residuals inside the NHs was decreased by the evaporation of $\mathrm{Au}$ and $\mathrm{Ga}$ while with the increment of annealing duration 
up to $7200 \mathrm{~s}$ the amount of residual was further decreased. Based on the EDS elemental characterization of spectra, similarly, the $\mathrm{Au} \mathrm{M \alpha l}$ peak $(2.12 \mathrm{keV})$ for the duration variation series was gradually decreased: 6800 counts for 1800 s; 4200 counts for 3600 s; 3900 counts for $7200 \mathrm{~s}$. The EDS spectra implies that the Au residual is gradually reduced along with the increased annealing duration and temperature due to the Au-Ga evaporation. In terms of dimensional variation of NHs: by increasing the annealing duration from 1800 to $3600 \mathrm{~s}$, the depth of NHs got a drastic increment from 221.8 to $811.1 \mathrm{~nm}$. From 3600 and $7200 \mathrm{~s}$, however, the diameter was slightly increased from 811.1 to $813.2 \mathrm{~nm}$ but depth was remained approximately constant $\sim 40.5 \mathrm{~nm}$. And in context of NHs shape, the longduration annealing made the edge cracked-like and irregular, as presented in Figure 9d,e. With $50 \mathrm{~nm} \mathrm{Au}$ annealed at $950{ }^{\circ} \mathrm{C}$ for $1800 \mathrm{~s}$, the large size Au NPs were drill down in NHs as clearly shown in Figure 9(f). Similarly, comparatively large and slightly drilling Au NPs were observed with $100 \mathrm{~nm}$ of Au deposition. (See in supporting information Figure S8)

\section{- CONCLUSION}

In summary, we have successfully fabricated porous $\mathrm{GaN}$ from $\mathrm{GaN}$ (0001) epilayer grown on sapphire by a thermal annealing using Au NPs as catalyst. Firstly, Au NPs were fabricated by the annealing of Au deposited GaN based on the Volmer-Weber growth model and these Au NPs were utilized as catalyst for the fabrication of NHs. The porous GaN was fabricated based on the concurrent phenomena of several processes. Due to the significantly enhanced GaN decomposition at the interface of NPs and GaN along with the absorption of Ga by the Au NPs, the formation of Au-Ga alloy NPs, release of $\mathrm{N}_{2}$ gas, penetration of NPs, evaporation of Au-Ga alloy simultaneously occurred, leading to the 
successful fabrication of NHs. We also studied the penetration depth of the NHs was enhanced with the increased annealing temperature and duration. The average penetration depth of NHs was increased by 22.03 times from 58 to $1336 \mathrm{~nm}$, the diameter 22.85 times from 35 to $835 \mathrm{~nm}$. As the same time, the density of NHs was reduced by 525 times from $8.4 \times 10^{11}$ to $1.6 \times 10^{9} \mathrm{~cm}^{-2}$. Furthermore, with the systematic variation of Au deposition amount, the evolution of NHs was precisely controlled in terms of the size, density and shape of the pores (nanoholes, NHs).

\section{- ASSOCIATED CONTENT}

\section{Supporting information}

This material is available free of charge via the Internet at http://pubs.acs.org.

\section{AUTHOR INFORMATION}

\section{Corresponding Author}

*e-mail: jihoonleenano@gmail.com

\section{Notes}

The authors declare no competing financial interest.

\section{- ACKNOWLEDGEMENTS}

This project was supported by the National Research Foundation of South Korea (no. 20110030079 and 2016R1A1A1A05005009), the National Science Foundation of USA (no. 
US/NSF/DMR1309989) and in part by the research grant of Kwangwoon University in 2016. 


\section{— REFERENCES}

(1) Diaz, D. J.; Williamson, T. L.; Adesida, I.; Bohn, P. W.; Molnar, R. J. J. Vac. Technol. B 2002, 20, 2375.

(2) Li, X.; Kim, Y. W.; Bohn, P. W.; Adesida, I. Appl. Phys. Lett. 2002, 80, 980.

(3) Tseng, W. J.; van Dorp, D. H.; Lieten, R. R.; Vereecken, P. M.; Borghs, G. J. Phys. Chem. C 2014, 118, 29492.

(4) Hartono, H.; Soh, C. B.; Chua, S. J.; Fitzgerald, E. A. Phys. Stat. Sol. (b) 2007, 244, 1793.

(5) Yam, F. K.; Hassan, Z.; Chuah, L. S.; Ali, Y. P. Appl. Surf. Sci. 2007, 253, 7429.

(6) Alshehri, B.; Lee, S. M.; Kang, J. H.; Gong, S. H.; Ryu, S. W.; Cho, Y. H.; Dogheche, E. Appl. Phys. Lett. 2014, 105, 051906.

(7) Wang, X. Z.; Yu, G. H.; Lin, C. T.; Cao, M. X.; Gong, H.; Qi, M.; Li, A. Z. Solid State Commun. 2010, 150, 168.

(8) Lee, J. H.; Lee, B.; Kang, J. H.; Lee, J. K.; Ryu, S. W. Thin Solid Films 2012, 525, 84.

(9) Yam, F. K.; Hassan, Z. Appl. Surf. Sci. 2007, 253, 9525.

(10) Ramizy, A.; Hassan, Z.; Omar, K. Sensor Actuat. B-Chem. 2011, 155, 699.

(11) Soh, C. B.; Tay, C. B.; Tan, R. J.; Vajpeyi, A. P.; Seetoh, I. P.; Ansah-Antwi, K. K.; Chua, S. J. J. Phys. D Appl. Phys. 2013, 46, 365102.

(12) Lee, K. J.; Kim, S. J.; Kim, J. J.; Hwang, K.; Kim, S. T.; Park, S. J. Opt. Express 2014, 22, A1164.

(13) Soh, C. B.; Liu, W.; Hartono, H.; Ang, N. S.; Chua, S. J.; Chow, S. Y.; Tay, C. B.; Vajpeyi, A. P. Appl. Phys. Lett. 2011, 98, 191906.

(14) Hartono, H.; Soh, C. B.; Chow, S. Y.; Chua, S. J.; Fitzgerald, E. A. Appl. Phys. Lett. 
2007, 90, 171917.

(15) Ghosh, B. K.; Tanikawa, T.; Hashimoto, A.; Yamamoto, A.; Ito, Y. J. Cryst. Growth 2003, 249,422 .

(16) Soh, C. B.; Hartono, H.; Chow, S. Y.; Chua, S. J.; Fitzgerald, E. A. Appl. Phys. Lett. $\mathbf{2 0 0 7}, 90,3112$.

(17) Schubert, M. F.; Chhajed, S.; Kim, J. K.; Schubert, E. F.; Koleske, D. D.; Crawford, M. H.; Lee, S. R.; Fischer, A. J.; Thaler, G.; Banas, M. A. Appl. Phys. Lett. 2007, 91, 231114.

(18) Nie, B.; Duan, B. K.; Bohn, P. W. ACS Appl. Mater. Inter. 2013, 5, 6208.

(19) Diaz, D. J.; Williamson, T. L.; Adesida, I.; Bohn, P. W.; Molnar, R. J. J. Appl. Phys. 2003, 94, 7526.

(20) Tseng, W. J.; van Dorp, D. H.; Lieten, R. R.; Vereecken, P. M.; Borghs, G. J. Phys. Chem. C 2014, 118, 29492.

(21) Schwartzkopf, M.; Buffet, A.; Korstgens, V.; Metwalli, E.; Schlage, K.; Benecke, G.; Perlich, J.; Rawolle, M.; Rothkirch, A.; Heidmann, B.; Herzog, G.; Muller-Buschbaum, P.; Rohlsberger, R.; Gehrke, R.; Stribeck, N.; Roth, S. V. Nanoscale 2013, 5, 5053.

(22) Chen, Z.; Lu, D.; Yuan, H.; Han, P.; Liu, X., Li, Y., Wang, X.; Lu, Y.; Wang, Z. A. J. Cryst. Growth 2002, 235, 188.

(23) Kalsin, A. M.; Fialkowski, M.; Paszewski, M.; Smoukov, S. K.; Bishop, K. J. M.; Grzybowski, B. A. Science 2006, 312, 420.

(24) Compton, O. C.; Osterloh, F. E. J. Am. Chem. Soc. 2007, 129, 7793.

(25) Xue, Q.; Xue, Q. Z.; Bakhtizin, R. Z.; Hasegawa, Y.; Tsong, I. S. T.; Sakurai, T. Phys. Rev. Lett. 1999, 82, 3074. 
(26) Pisch, A.; Schmid-Fetzer, R. J. Cryst. Growth 1998, 187, 329.

(27) Groh, R.; Gerey, G.; Bartha, L.; Pankove, J. I. Phys. Stat. Sol. (a) 1974, 26, 353.

(28) L'vov, B. V.; Thermochim. Acta 2000, 360, 85.

(29) Fernandez-Garrido, S.; Koblmuller, G.; Calleja, E.; Speck, J. S. J. Appl. Phys. 2008, $104,033541$.

(30) Koleske, D. D.; Wickenden, A. E.; Henry, R. L.; Culbertson, J. C.; Twigg, M. E. J. Cryst. Growth 2001, 223, 466.

(31) Hocevar, M.; Immink, G.; Verheijen, M.; Akopian, N.; Zwiller, V.; Kouwenhoven, L.; Bakkers, E. Nat. commun. 2012, 3, 1266.

(32) Hou, W. C.; Chen, L. Y.; Tang, W. C.; Hong, F. C. Cryst. Growth Des. 2011, 11, 990.

(33) Liu, J.; Guo, C.; Li, C.; Du, Z. J. Alloy. Compd. 2010, 508, 62.

(34) Meng, G.; Yanagida, T.; Kanai, M.; Suzuki, M.; Nagashima, K.; Xu, B.; Zhuge, F.; Klamchuen, A.; He, Y.; Rahong, S.; Kai, S.; Kawai, T. Phys. Rev. E 2013, 87, 012405.

(35) Choi, W. K.; Liew, T. H.; Chew, H. G.; Zheng, F.; Thompson, C. V.; Wang, Y.; Hong, M. H.; Wang, X. D.; Li, L.; Yun, J. A. Small 2008, 4, 330.

(36) Campbell, C. T.; Parker, S. C.; Starr, D. E. Science 2002, 298, 811.

(37) Barnard, A. S.; Chen, Y. J. Mater. Chem. 2011, 21, 12239.

(38) Tao, A. R.; Habas, S.; Yang, P. Small 2008, 4, 310.

(39) Li, C. R.; Lu, N. P.; Mei, J.; Dong, W. J.; Zheng, Y. Y.; Gao, L.; Tsukamoto, K.; Cao, Z. X. J. Cryst. Growth 2011, 314, 324. 


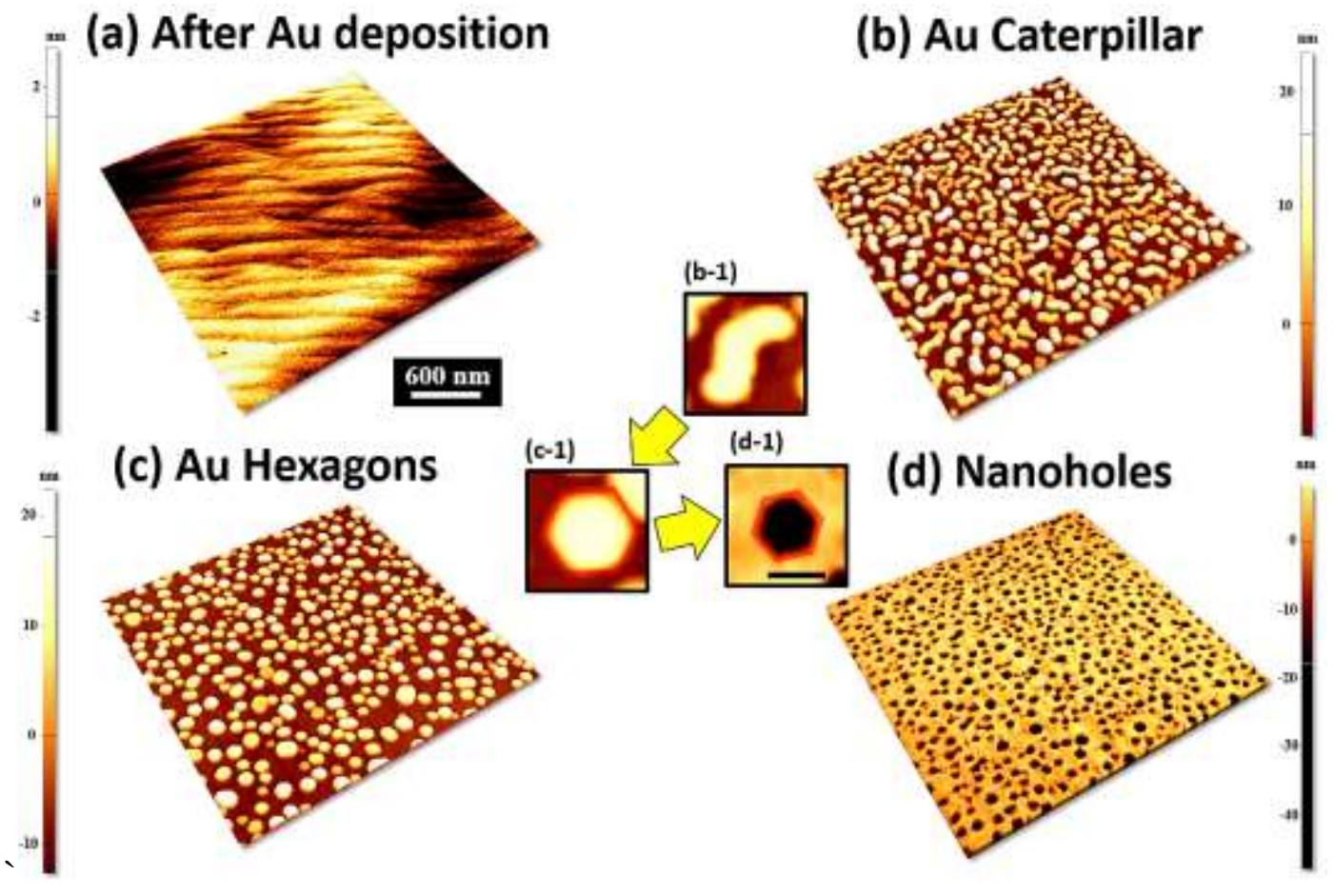

Figure 1. Evolution of nanostructures fabricated at various temperatures on $\mathrm{GaN}(0001)$ with $3 \mathrm{~nm} \mathrm{Au}$ deposition for 450 s. (a) Au deposition on GaN (0001). (b) Au caterpillars at $450{ }^{\circ} \mathrm{C}$. (c) Au hexagons at $750^{\circ} \mathrm{C}$. (d) Nanoholes at $900{ }^{\circ} \mathrm{C}$. Panels a - d AFM sideviews of $3 \times 3 \mu \mathrm{m}^{2}$. Panels b- $1-\mathrm{d}-1$ AFM top-views of $200 \times 200 \mathrm{~nm}^{2}$ and the black scale bar is $100 \mathrm{~nm}$. 
(a) Bare surface
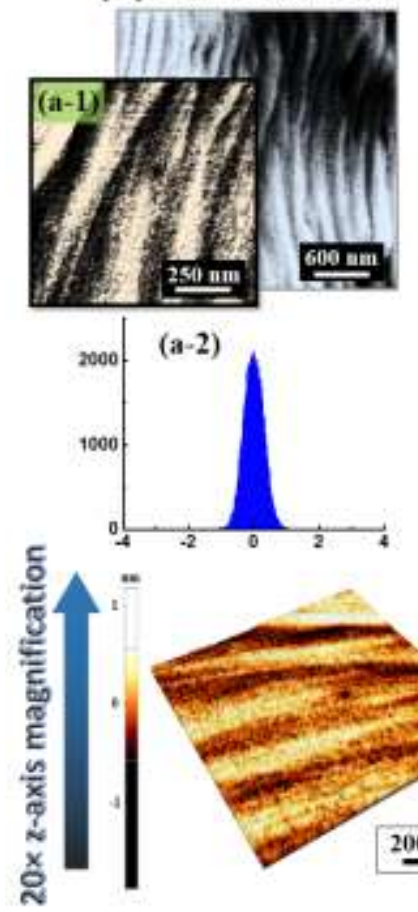

(b) Annealing at $800^{\circ} \mathrm{C}$
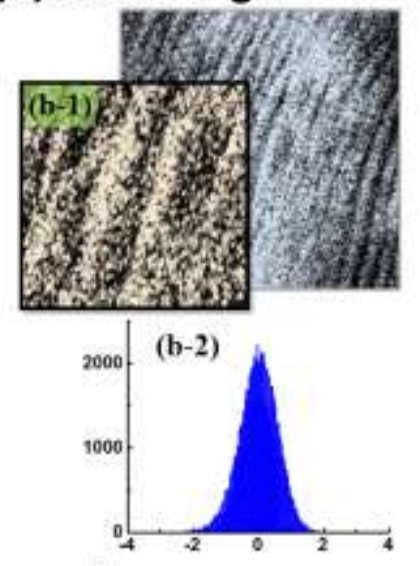

=

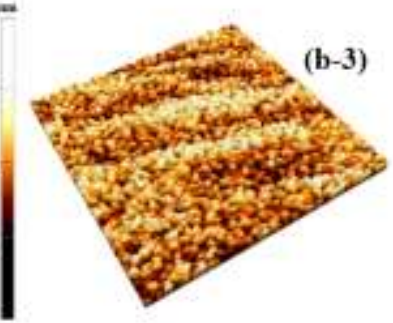

(c) Annealing at $900^{\circ} \mathrm{C}$
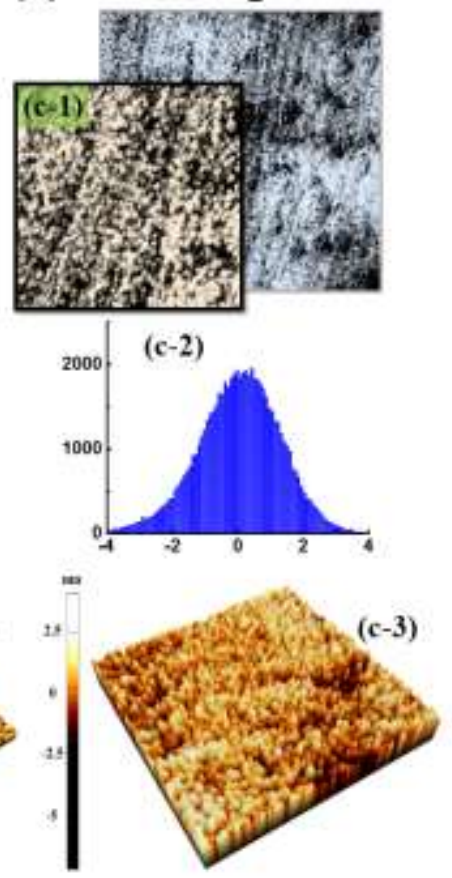

Figure 2. Surface decomposition of GaN (0001) at high temperature. (a) Bare GaN surface. (b) $\mathrm{GaN}$ annealed at $800{ }^{\circ} \mathrm{C}$ for 3600 s. (c) $\mathrm{GaN}$ annealed at $900{ }^{\circ} \mathrm{C}$ for 3600 s. Panels a $\mathrm{c}$ are $3 \times 3 \mu \mathrm{m}^{2}$ AFM top-views. Panels a-1 $-\mathrm{c}-1$ are $1 \times 1 \mu \mathrm{m}^{2}$. Panels a-2 $-\mathrm{c}-2$ Height distribution histograms. Panels a-3 - c-3 AFM side-views of $1 \times 1 \mu \mathrm{m}^{2}$. In Panels b - c, tiny Ga clusters are observed due to the vertical decomposition of nitrogen. 

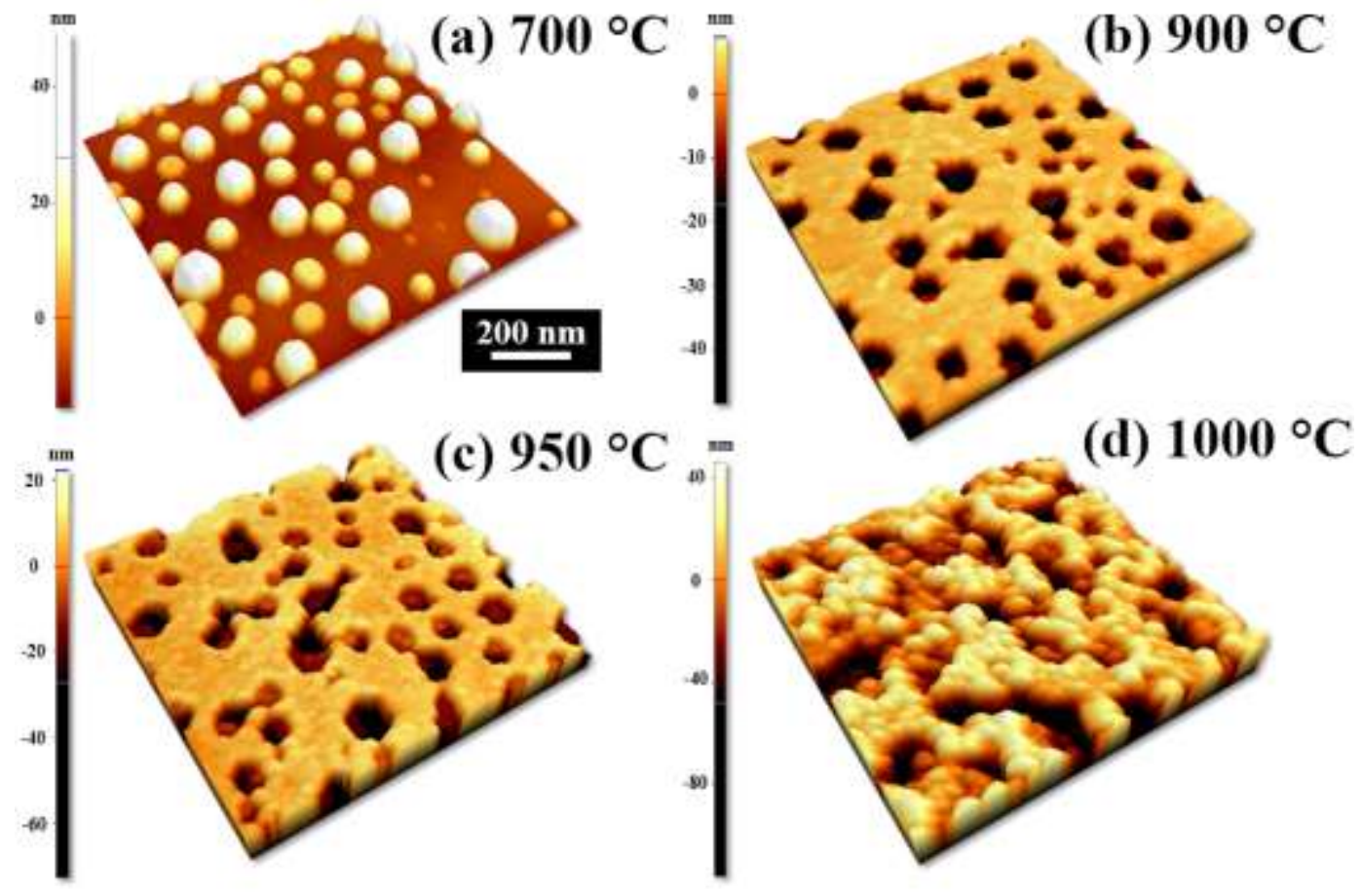

(d) $1000{ }^{\circ} \mathrm{C}$
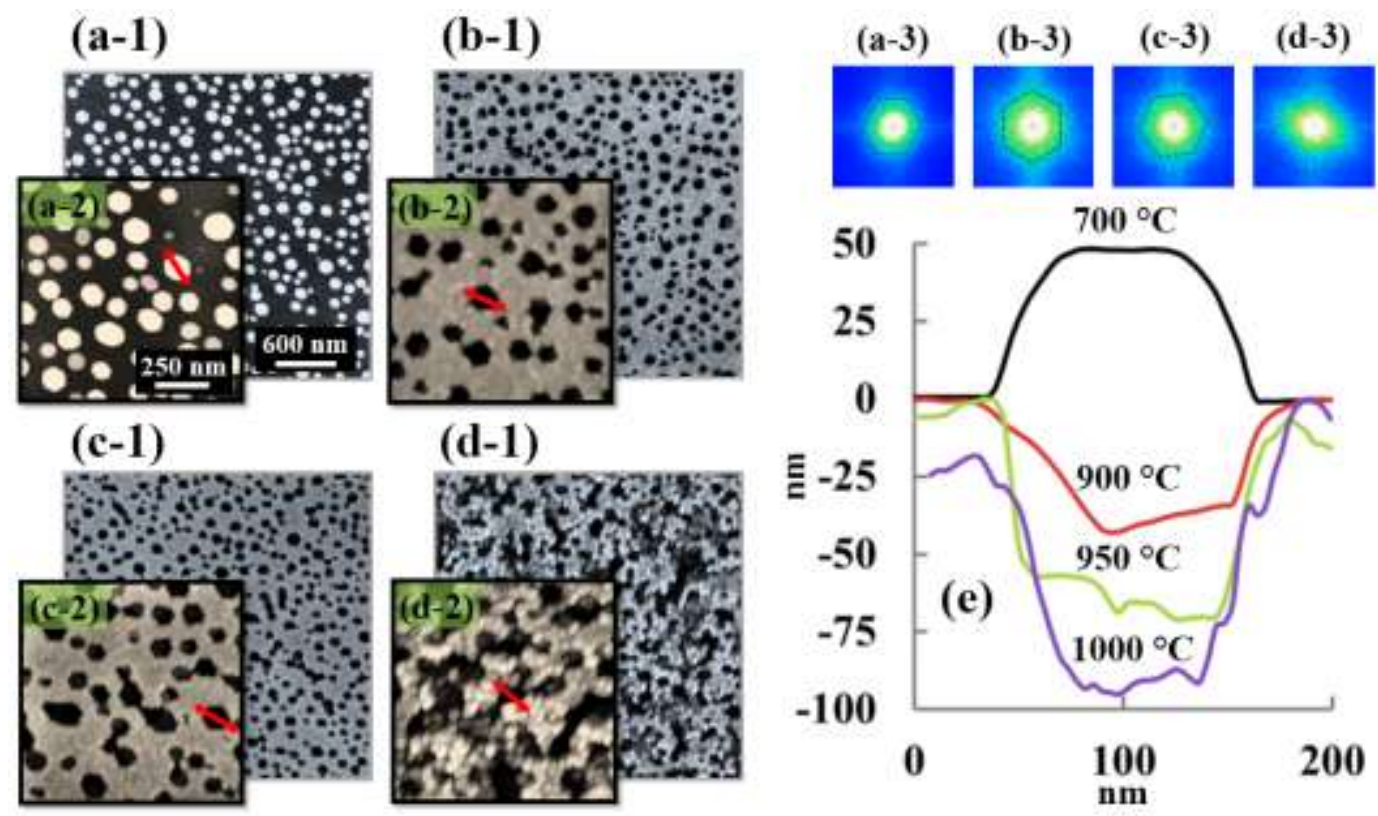

Figure 3. Temperature induced evolution from Au nanoparticles at (a) $700{ }^{\circ} \mathrm{C}$ to nanoholes at (b) 900 , (c) 950 and (d) $1000{ }^{\circ} \mathrm{C}$ for $450 \mathrm{~s}$ with $4 \mathrm{~nm}$ deposition on $\mathrm{GaN}$ (0001). Panels $\mathrm{a}-\mathrm{d}$ AFM side-views of $1 \times 1 \mu \mathrm{m}^{2}$. Panels a- $1-\mathrm{d}-1$ AFM top-views of $3 \times 3 \mu \mathrm{m}^{2}$. Panels a-2 - d-2 AFM top-views of $1 \times 1 \mu \mathrm{m}^{2}$. Panels a-3 - d-3 Fourier filter transform (FFT) power spectra of corresponding samples. (e) Multi cross-sectional line-profiles of the locations indicated by the red arrows in Panels a-2 - d-2. 

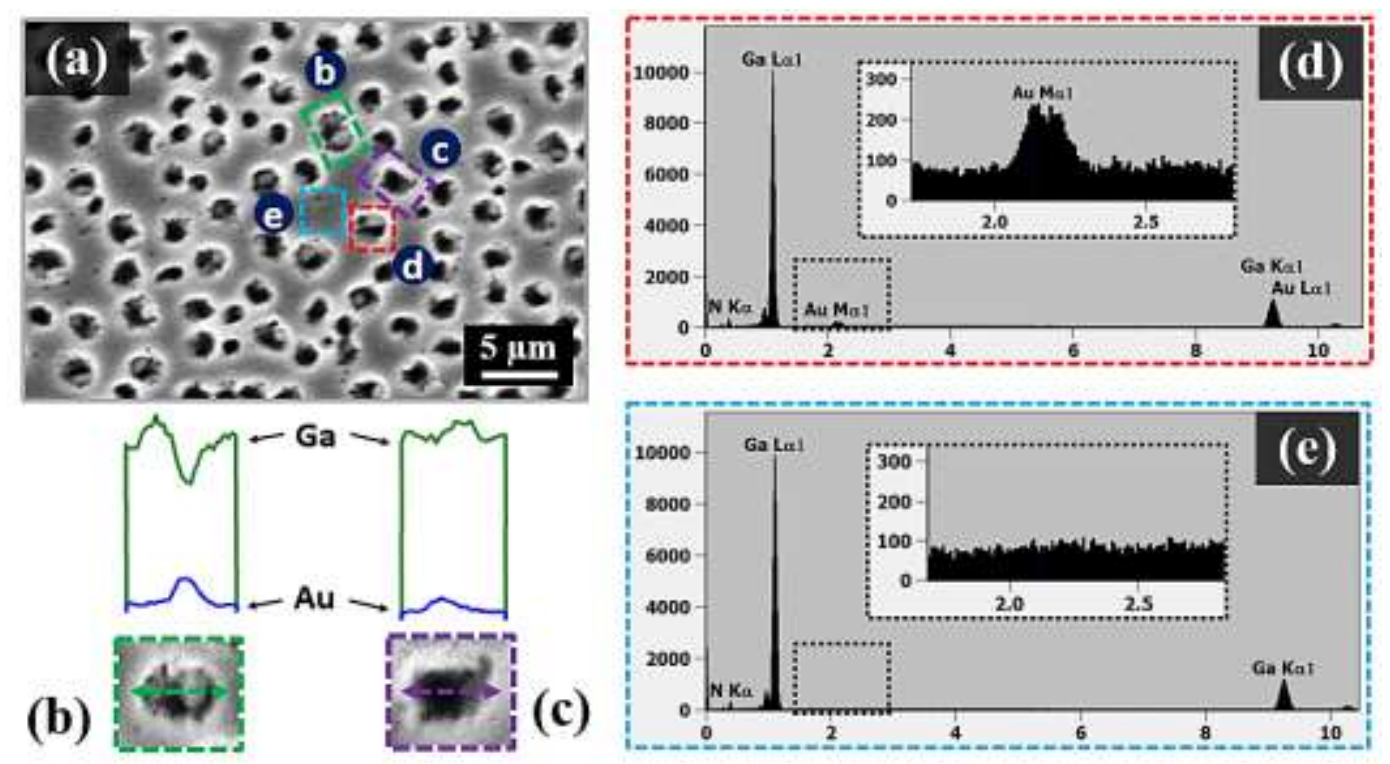

Figure 4. Energy-dispersive X-ray spectroscopy spectral analysis of nanoholes fabricated by the annealing at $900{ }^{\circ} \mathrm{C}$ for $3600 \mathrm{~s}$ with $25 \mathrm{~nm} \mathrm{Au} \mathrm{GaN} \mathrm{(0001).} \mathrm{(a)} \mathrm{SEM} \mathrm{image.} \mathrm{(b} \mathrm{-} \mathrm{c)}$ $\mathrm{Ga}$ and $\mathrm{Au}$ EDS line-profiles of two nanoholes indicated by (b) green and (c) purple rectangles in (a). (d-e) EDS spectra indicated by (d) red and (e) blue rectangles in (a). 


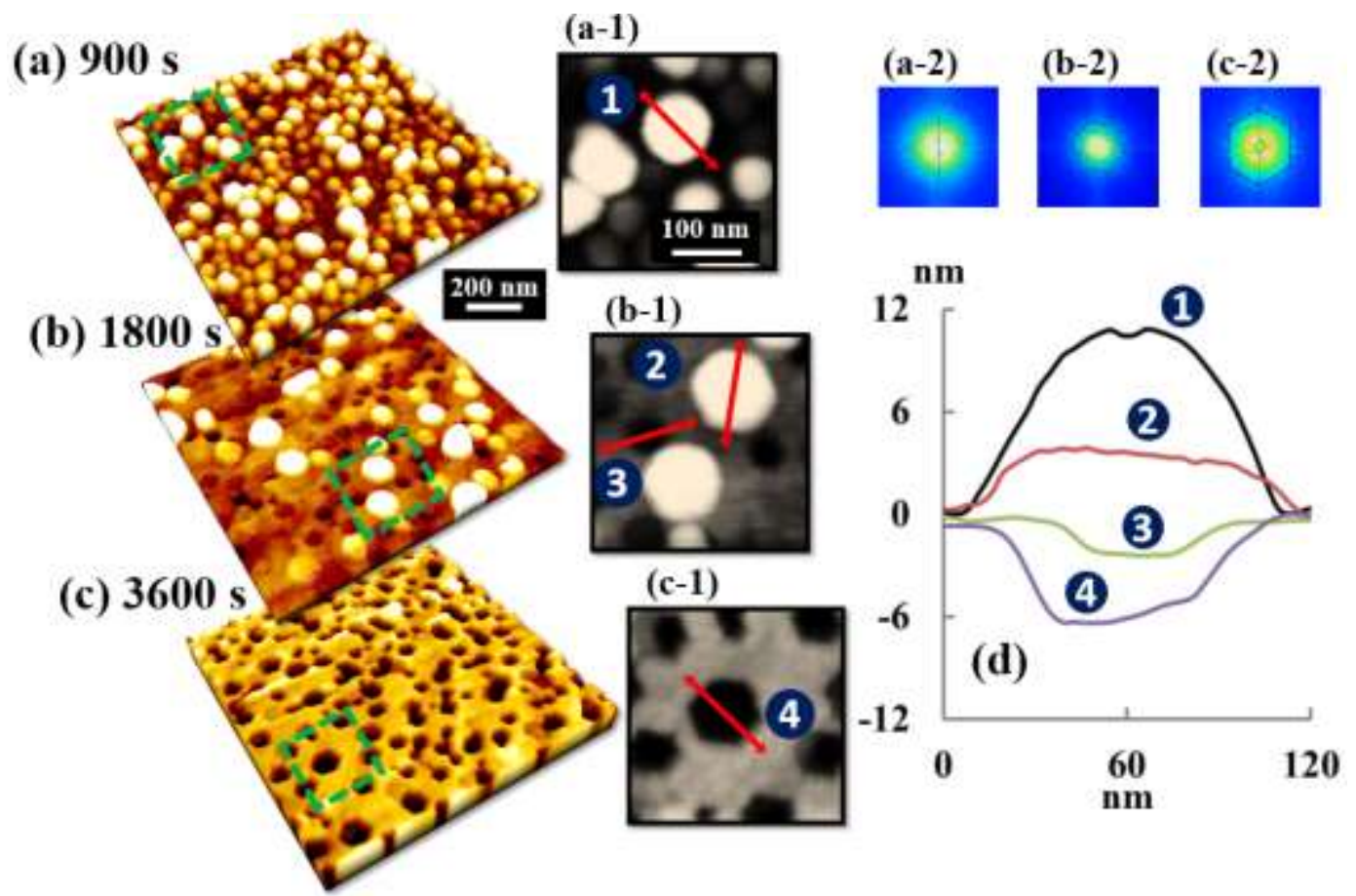

Figure 5. Annealing duration dependent evolution of Au hexagons into the nanoholes at $800{ }^{\circ} \mathrm{C}$ with $3 \mathrm{~nm} \mathrm{Au}$ deposition. (a) Au hexagons (b) Au hexagons and nanoholes (c) Nanoholes. Panels a $-\mathrm{c}$ AFM side-views of $1 \times 1 \mu \mathrm{m}^{2}$. Panels a- $1-\mathrm{c}-1$ typical region marked by the green rectangle in Panels a - c. Panels a-2 - c-2 FFT power spectra. (d) Multi cross-sectional line-profiles indicated by the red arrows in Panels a-1 - c-1. 


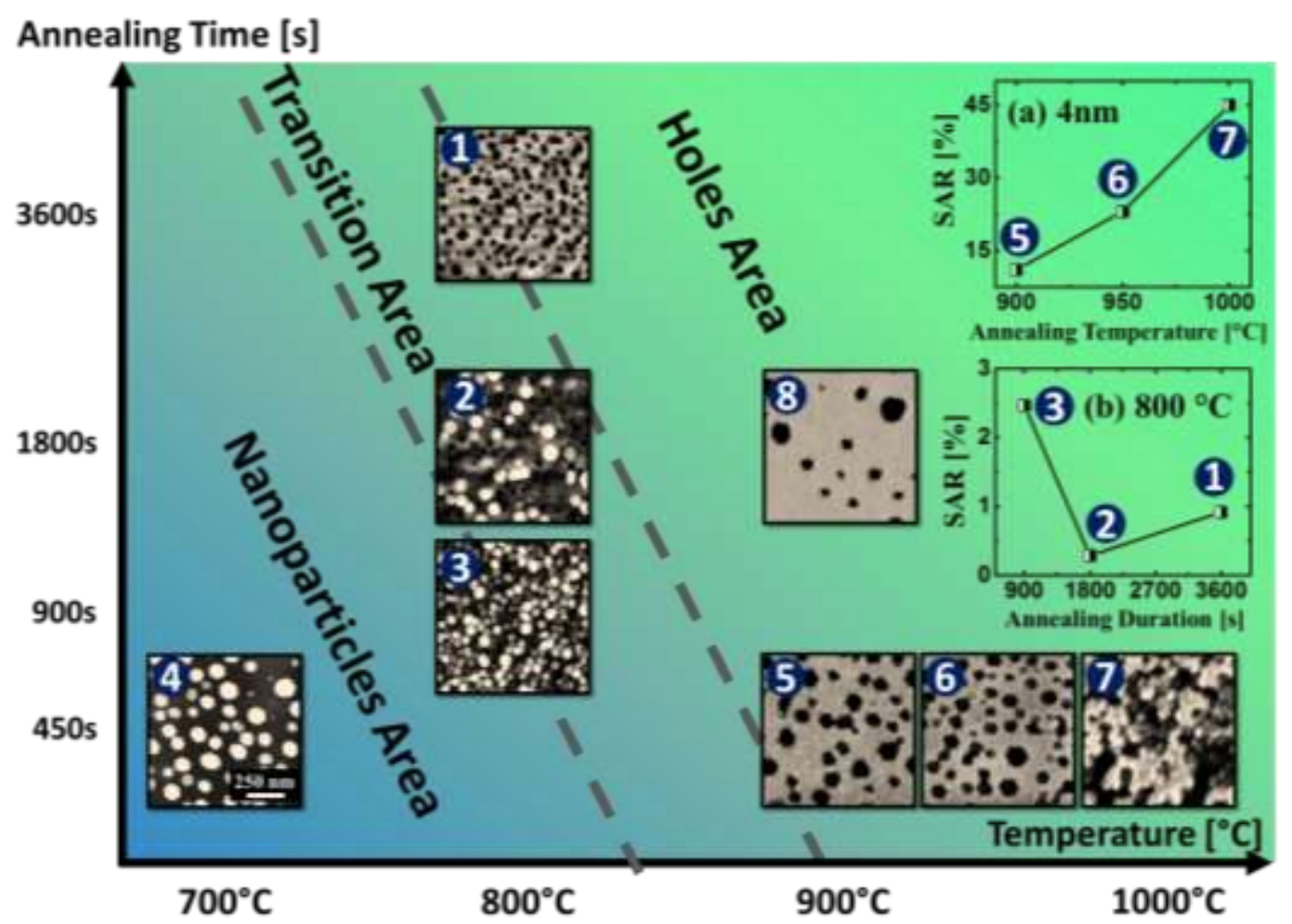

Figure 6. Map of the fabrication of nanoholes with the variation of annealing temperature versus annealing duration. (a) Surface area ratio (SAR) of the samples fabricated with 4 $\mathrm{nm}$ Au deposition at various temperatures. (b) SAR of samples fabricated at $800{ }^{\circ} \mathrm{C}$ with 3 $\mathrm{nm}$ deposition with the variation of annealing duration. 


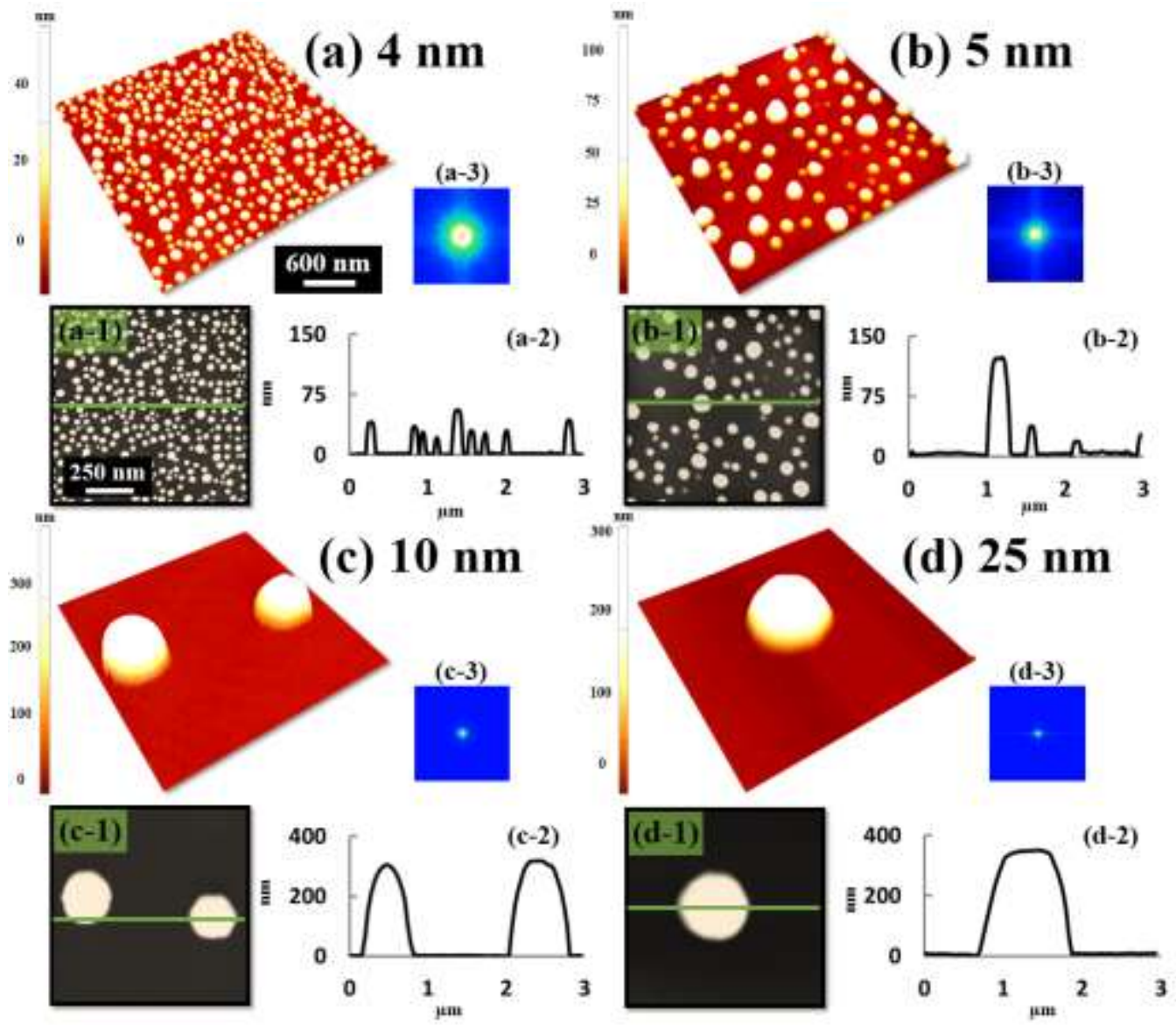

Figure 7. Control of $\mathrm{Au}$ nanoparticles by the variation of $\mathrm{Au}$ deposition amount on $\mathrm{GaN}$ (0001). (a) Au nanoparticles fabricated at $700^{\circ} \mathrm{C}$ for $900 \mathrm{~s}$, (b) at $750{ }^{\circ} \mathrm{C}$ for $900 \mathrm{~s}$, (c) at $700^{\circ} \mathrm{C}$ for $450 \mathrm{~s}$, and (d) at $750^{\circ} \mathrm{C}$ for $450 \mathrm{~s}$. Panels a - d AFM top-views of $3 \times 3 \mu \mathrm{m}^{2}$. Panels a-1 - d-1 AFM top-views of $3 \times 3 \mu \mathrm{m}^{2}$. Panels a- $-\mathrm{g}-2$ The related line-profiles indicated with green lines. Panels a-3 - g-3 Corresponding FFT power spectra. 

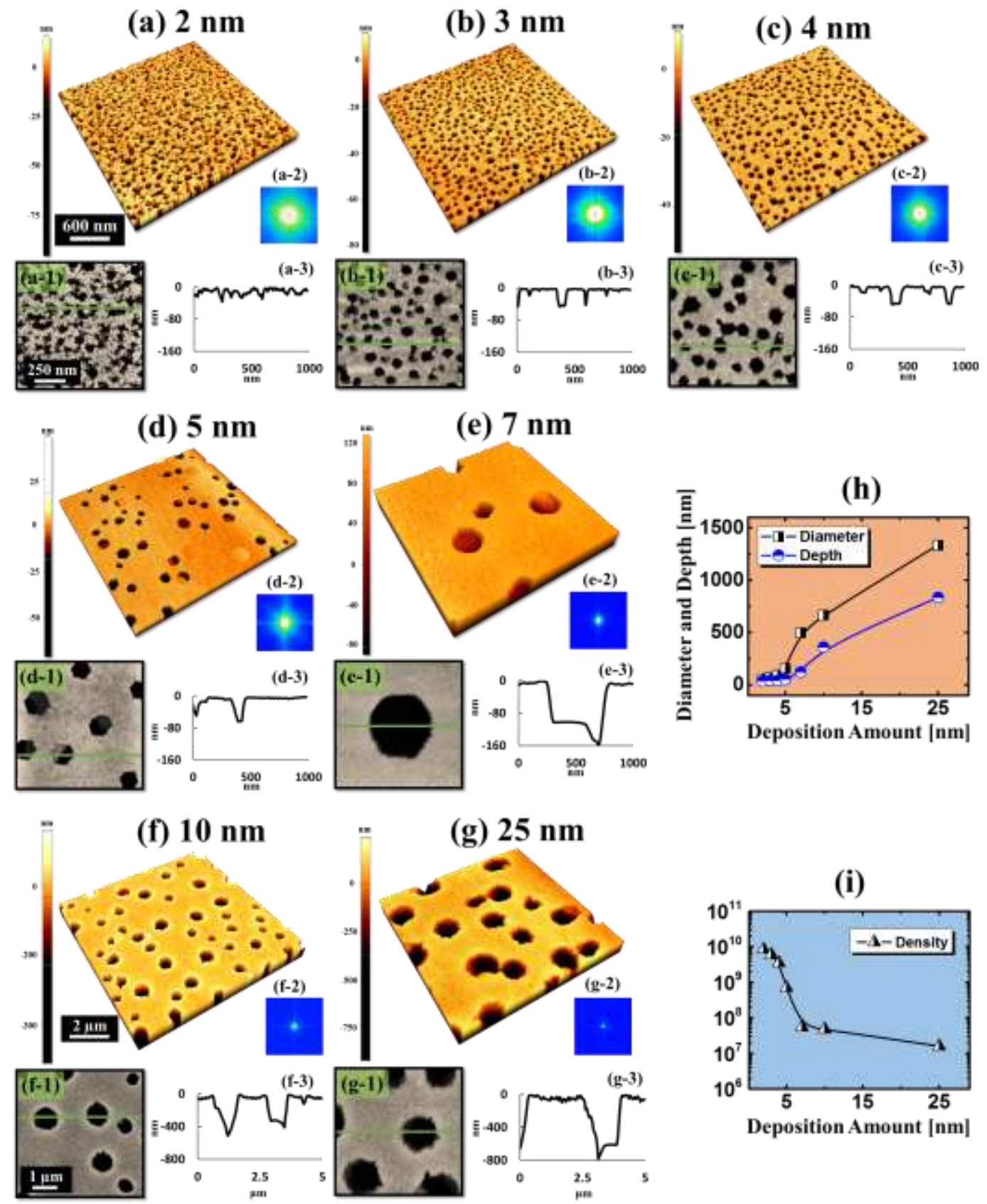

(i)

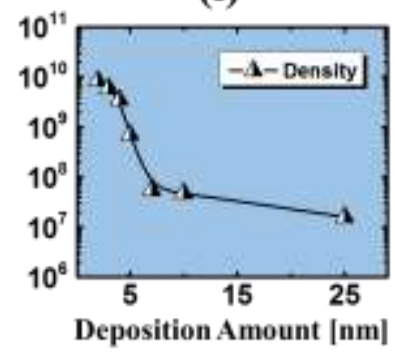

Figure 8. Control of size and density of nano-holes by the variation of Au deposition amount from 2 to $25 \mathrm{~nm}$ as labeled. (a - e) AFM side-views of $3 \times 3 \mu \mathrm{m}^{2}$. Panels a- $1-\mathrm{e}-$ 1 Detailed AFM top-views of $1 \times 1 \mu \mathrm{m}^{2}$. (f $-\mathrm{g}$ ) AFM side-views of $10 \times 10 \mu \mathrm{m}^{2}$. Panels $\mathrm{f}-1-\mathrm{g}-1$ Detailed AFM top-views of $5 \times 5 \mu \mathrm{m}^{2}$. Panels a- $2-\mathrm{g}$-2 Corresponding FFT power spectra. Panels a-3 - g-3 The related line-profiles indicated with green lines. (h) Corresponding average depth and diameter versus Au deposition amount. (i) Density of nanoholes. 


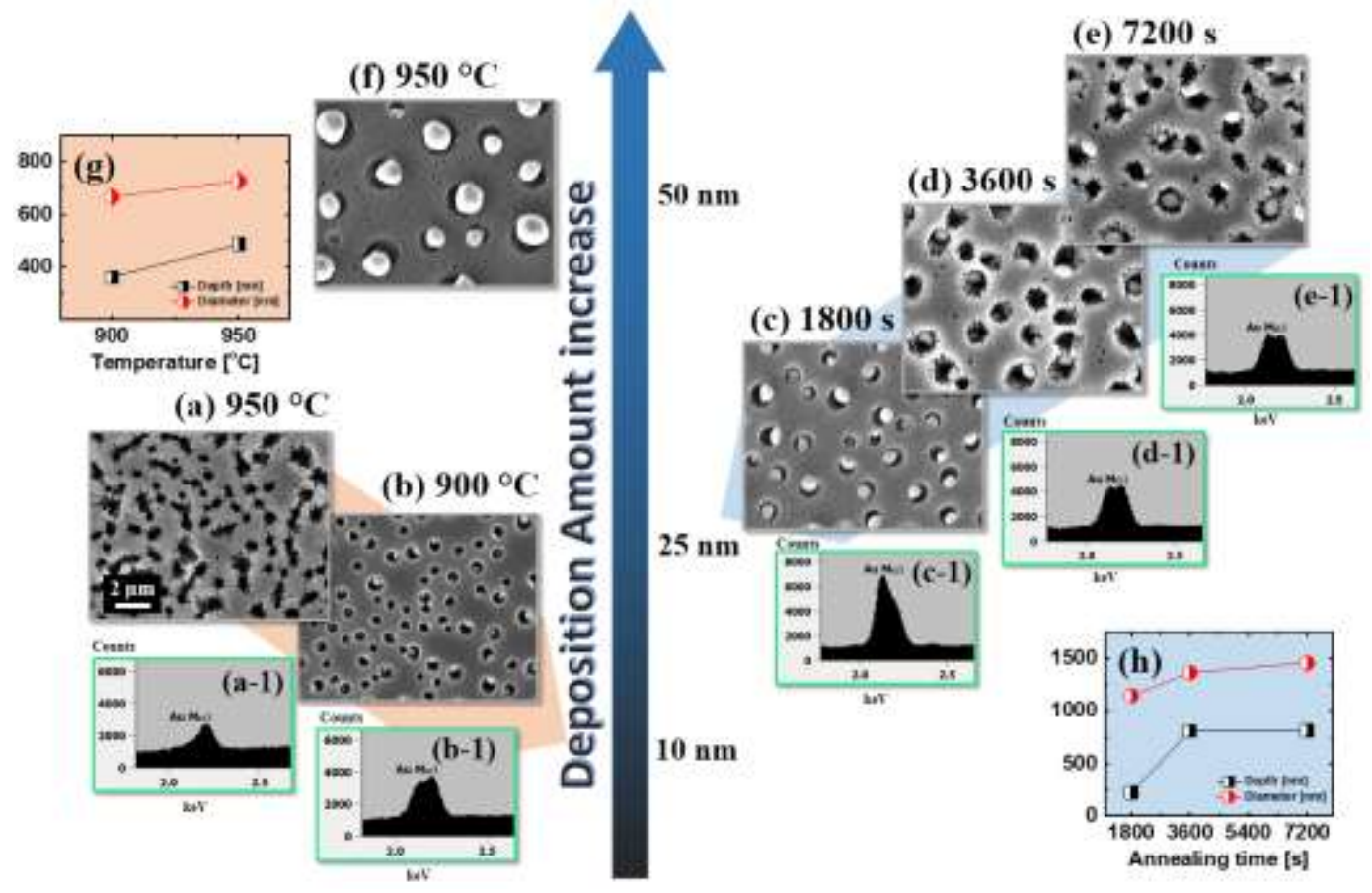

Figure 9. Temperature and duration effect on the fabrication of nanoholes with various $\mathrm{Au}$ deposition amounts as labeled. (a - b) $10 \mathrm{~nm}$ with annealing for $3600 \mathrm{~s}$. (c-e) $25 \mathrm{~nm}$ with annealing at $900{ }^{\circ} \mathrm{C}$. (f) $50 \mathrm{~nm}$ with annealing for $1800 \mathrm{~s}$. Panels a - f SEM images. Panels a-1 - e-1 EDS spectra of corresponding samples in the energy range from 2 to $2.5 \mathrm{keV}$. (g - h) Related depth and diameter of holes, acquired from the corresponding AFM images. 


\section{For Table of Contents Use Only}

\section{Graphical Abstract}

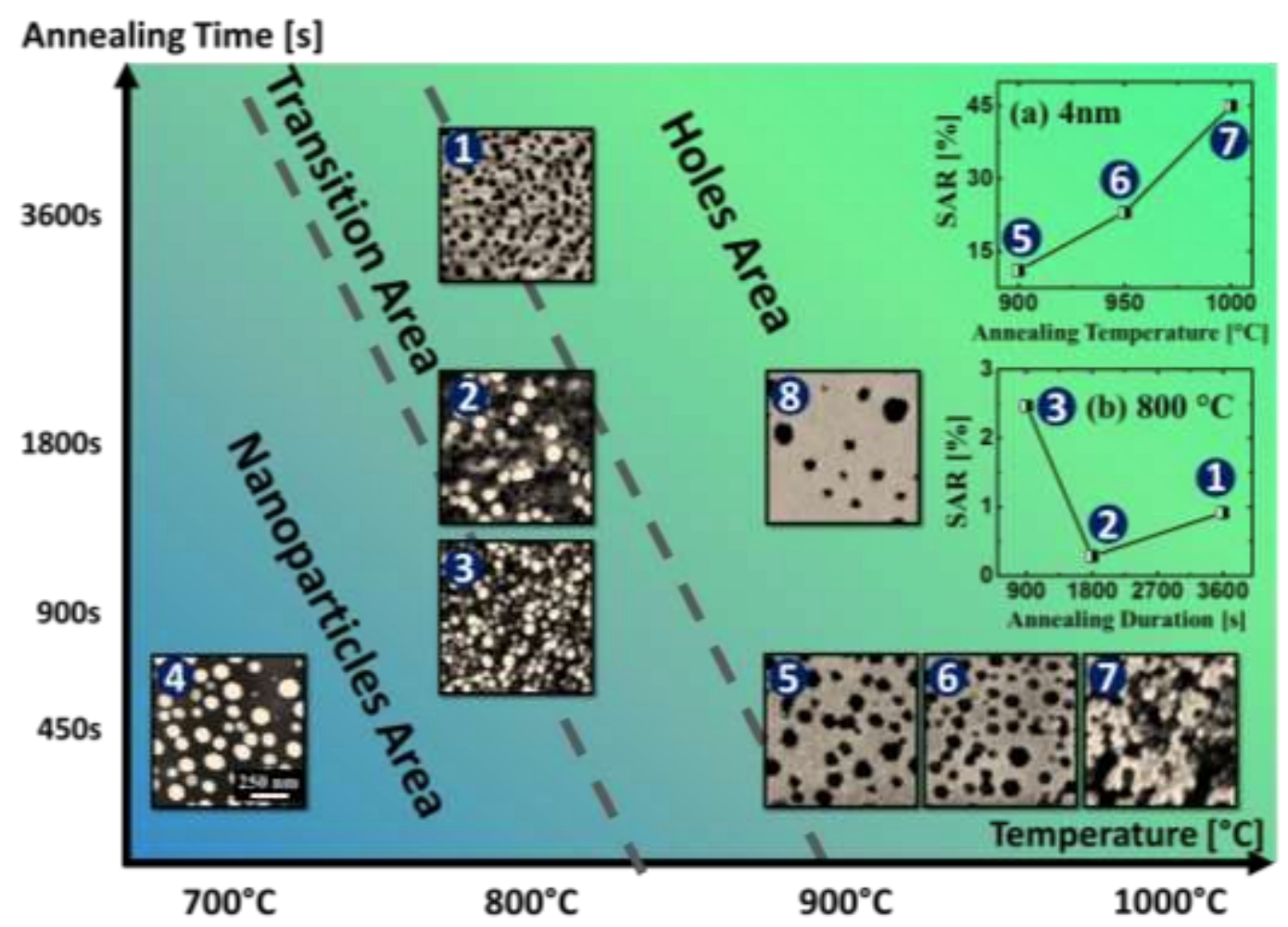

\section{Synopsis}

"The fabrication of nanoholes on GaN by the thermal annealing at elevated temperature using Au nanoparticles as catalyst, has been demonstrated. The shape, size and density of the nanoholes were precisely controlled based on the systematic variation of annealing temperature, time and deposition amount." 